\title{
The power of peptides: Cell-penetrating and signal peptide sequences for precision targeting of probes in imaging and sensing in live cells
}

\author{
Aisling Byrne, Christopher Burke, Tia Keyes, Aaron Martin
}

Dublin City University, Ireland

https://doi.org/10.17952/35EPS.2018.323

Cell-penetrating peptides (CPPs) are short peptide sequences, typically less than 30 amino acids long that are effective in carrying appended impenetrable cargo across the plasma membrane. The most commonly studied CPPs include Transactivator of transcription(TAT), Penetratin, a domain from Antennapedia protein and polyarginines, which are cationic, and are believed to drive transport through endocytosis. Their primary application to date has been to transport therapeutic agents across the cell membrane in clinical applications and have also increasingly been applied to driving luminescent probes into cells for imaging applications.

The main advantage of using CPPs in cell imaging and sensing is that they promote and facilitate cellular uptake of molecules and can do so at low concentrations, without damage to the membrane and with minimal cytotoxicity. A drawback is that endocytosis is the transport mechanism, this can lead to isolation of the cargo in endosomes rather than released into the cytoplasm or organelle of interest. We have exploited the following $\mathrm{CPPs}$ based on sequences found in proteins in organelle membranes. In order to direct our $\mathrm{Ru}$ (II) probes for imaging and sensing to specific organelles in live cells we have also exploited signal peptide sequences such as the mitochondrial penetrating peptide (MPP) sequence FrFKFrFK ( $\mathrm{r}=\mathrm{d}$-arginine),[1] nuclear localising signal (NLS) sequence VQRKRQKLMP which is derived from the NF- $\kappa \mathrm{B}$ transcription factor,[2] and the endoplasmic reticulum (ER) localising sequence RQIKIWFQNRRMKWKK which is derived from the Drosophila transcription factor Antennepedia.[3] All $\mathrm{Ru}$ (II) parent complexes, i.e. without a peptide present, are membrane impermeable without the use of detergents or organic solvent. An advantage of Ruthenium polypyridyl complexes is their synthetic flexibility wherein the ligand can be altered in order to create the required response, for example, 1,10-phenanthroline(phen) or 4,7 diphen1,10-phenanthroline containing complexes are suitable for O2/ROS sensitivity, dipyridophenazine (dppz) promotes DNA binding/ intercalation and causes metal complex emission to switch off in water but switch on when bound and tetraazaphenathrene(tap) for phototoxic activity induced by photoinduced electron transfer to DNA. By combining our $\mathrm{Ru}(\mathrm{II})$ probes with $\mathrm{CPP}$ sequences we have created a library of probes capable of high precision targeting for imaging, including super-resolution STED imaging, and sensing in live cells.

\section{Measurement of $\mathrm{O}_{2}$ and ROS in the mitochondria of live cells}

By combining an oxygen sensitive Ruthenium probe with a mitochondrial penetrating peptide (MPP) sequence FrFKFrFK (Fig 1A), delivery of the $\mathrm{O}_{2}$ probe selectively to the mitochondria was achieved. Uptake is quick, with $\left[\left(\mathrm{Ru}(\mathrm{bpy})_{2} \text { phen-Ar }\right)_{2}-\mathrm{MPP}\right]^{7+}(70 \mu \mathrm{M})$ localising in the mitochondria after $2 \mathrm{~h}$. Using Antimycin A, a mitochondrial uncoupler, we were able to increase the $\mathrm{O}_{2}$ levels in the mitochondria, and measure the concentrations based on the luminescent lifetime using Luminescent Lifetime Imaging Microscopy (LLIM). The lifetime data was collected every 10 minutes following Antimycin A treatment and a decrease in lifetime was observed, consistent with rising oxygen levels as mitochondrial metabolism decreases, and $\mathrm{O}_{2}$ quenches the emission of the complex. The emission lifetime decrease was observed to fall below the value expected for $\mathrm{O}_{2}$ saturation, indicating that ROS was produced at the mitochondria which was more effective in quenching the complex luminescence.[4]

\section{Targeting and Imaging mtDNA in live cells}

Using the same MPP sequence (Fig 1A) but changing the ruthenium probe to target DNA using the dppz 'light-switch' ligand results in a mitochondrial penetrating complex $[\mathrm{Ru}(\mathrm{dppz}-\mathrm{FrFKFrFK})]^{5+}$, that was capable of binding and emitting from mtDNA. Figure 1A (i) shows $[\mathrm{Ru}(\mathrm{dppz}-\mathrm{FrFKFrFK})]^{5+}$ (green) colocalised with MitoTracker Deep Red (red) (ii). Along the mitochondrial strands, bright punctate spots in yellow indicate binding of the probe to mtDNA (iii). As the dppz 'light-switch' ligand only emits when protected from water, we can attribute the bright emission to interactions with mtDNA. The luminescence lifetime data was also consistent 
with DNA binding, and protein or RNA binding could be excluded on this basis.[5]

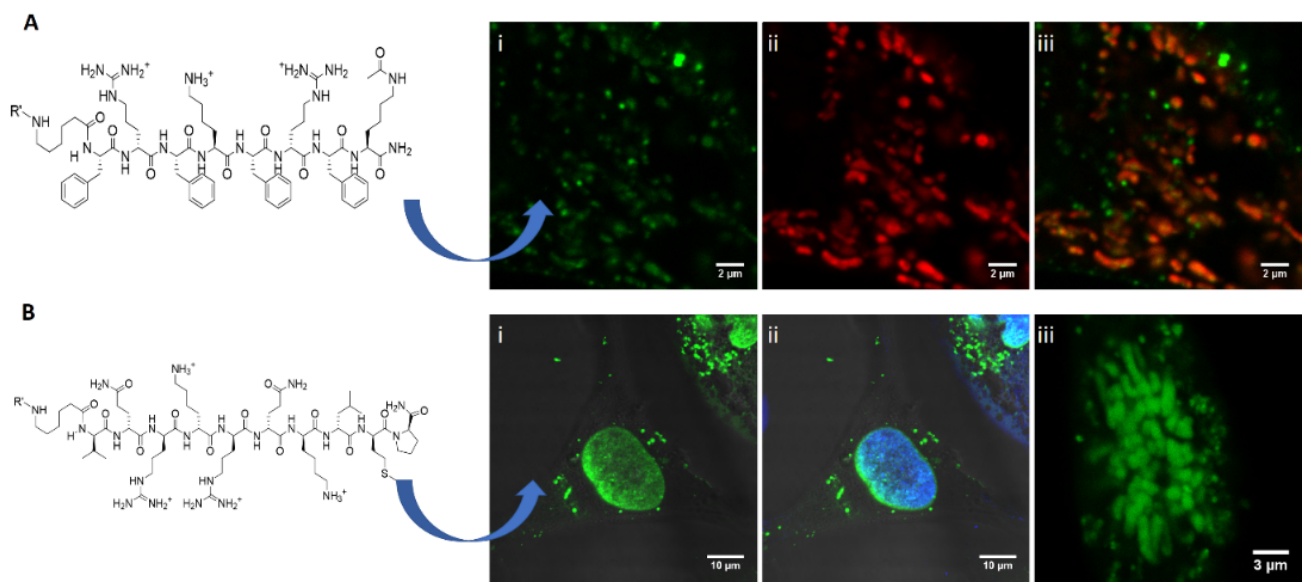

Figure 1: Structure of the mitochondrial penetrating peptide (MPP) sequence FrFKFrFK (A). $[R u(d p p z-F r F K F r F K)] 5+$ in the mitochondria of a live HeLa cell in green (i), the commercial probe MitoTracker Deep Red (ii), and their colocalisation in orange (iii). Structure of nuclear localising sequence $\operatorname{VQRKRQKLMP~(B).[Ru(dppz)(bpy)(bpy-Ar-NLS)]6+~in~the~nucleus~of~a~live~HeLa~cell~(i),~colocalised~with~}$ DAPI (ii), and it bound to nuclear chromosomes (iii).

\section{STED of Nuclear DNA and chromosomes during mitosis}

Using the DNA light-switch ligand we conjugated the nuclear localising sequence (NLS) VQRKRQKLMP (Fig 1B) to this complex resulting in $[\mathrm{Ru}(\mathrm{dppz})(\mathrm{bpy})(\mathrm{bpy}-\mathrm{Ar}-\mathrm{NLS})]^{6+}$. Our DNA targeting probe successfully crosses the cell membrane, penetrates the nuclear envelope (Fig 1B (ii)) and colocalises with DAPI (Fig 1B (ii)). Figure $1 \mathrm{~B}$ (iii) shows that $[\mathrm{Ru}(\mathrm{dppz})(\mathrm{bpy})(\mathrm{bpy}-\mathrm{Ar}-\mathrm{NLS})]^{6+}$ binds to nuclear chromosomes.[6] After directing $[\mathrm{Ru}(\mathrm{dppz})(\mathrm{bpy})(\mathrm{bpy}-\mathrm{Ar}-\mathrm{NLS})]^{6+}$ to the nucleus, we applied Stimulated Emission Depletion (STED), a super resolution imaging technique. The image contrast of the chromosomes is clearer and more resolved after applying the $660 \mathrm{~nm}$ STED depletion laser, compared to confocal, and the groove detail of the sister chromatids can be resolved.[6]

\section{STED of Endoplasmic Reticulum structures}

To target the endoplasmic reticulum (ER) we conjugated the endoplasmic reticulum directing signal peptide sequence RQIKIWFQNRRMKWKK to a Ru(II) complex which resulted in [(Ru(bpy) ${ }_{2}$-phen-Ar)-ER $]^{9+}$. $\left[\left(\mathrm{Ru}(\mathrm{bpy})_{2} \text {-phen-Ar)-ER }\right]^{9+}(70 \mu \mathrm{M})\right.$ successfully crosses the cell membrane, and localizes in the ER, which was confirmed using ER-Tracker Blue. In the confocal image, the ER appear as small, punctate spots. However, upon using the STED $660 \mathrm{~nm}$ depletion laser, the tubule structure of the smooth ER becomes clearly resolved. This is reflected in the FWHM of the ER, where it decreased from $532.44 \mathrm{~nm}$ to $226.66 \mathrm{~nm}$.[6] Overall, combining $\mathrm{CPP}$ or signal peptides with Ruthenium polypyridyl complexes is a powerful approach to imaging cells with super-resolution and can enable real time insights into metabolic changes at discrete organelles.

\section{References}

1 Ragin et al. Chem Biol. 2002, 9 ,943-948.

2 Hortonet al. Chemistry \& Biology. 2008, 15, 375-382. DOI 10.1016/j.chembiol.2008.03.015

3 Pietersz et al. Vaccine. 2001, 19, 1397-1405.

4 Martin et al. J. Am. Chem. Soc., 2014, 136, 15300 -15309. DOI: 10.1021/ja508043q

5 Burke et al. Angew.Chem. Int.Ed., 2018, 57,2420 -12424. DOI: 10.1002/anie.201806002

6 Byrne et al. Chem. Sci., 2016, 7, 6551. DOI: 10.1039/c6sc02588a 